Mathematical Modelling and Analysis, vol. 3, pp. 136-139

R.Čiegis (Ed)

(C) 1998 Technika

\title{
ON THE BLOOD GLUCOSE DYNAMICS MODELLING
}

\author{
M. MEILŪNAS \\ Vilnius Gediminas Technical University \\ Sauletekio 11, Vilnius, \\ Vilnius University, Universiteto 3, Vilnius \\ Klaipèda University, Manto 84, Klaipèda \\ E-mail:mecislovas.meilunas@.fm.vtu.lt
}

\begin{abstract}
Mathematical model of the glucose regulation is proposed and discussed. It is based only on the data, which are available in outpatient clinics. This model enables to predict the state of the patients health and to identificate important parameters of patients organism, which are urgent in diabetology.
\end{abstract}

\section{INTRODUCTION}

During the past decades a great number of the computer-based technologies have been developed to solve numerous problems of diabetes care. Data bases, expert systems, integrated patient management systems provide a wide range of instruments for everyday decision making in (outpatient clinics). We refere to [4] as to a state of the art overview.

In each software tool devoted to diabetes mellitus (DM) patient problems any idea of blood glucose level regulation modeling is embodied in direct or indirect way. Therefore the mathematical models in diabetology are a necessary elements even in theoretical studies as in computerized diabetes monitoring and treatment systems.

There are variuos ways to construct mathematical models in diabetology. The feature of mathematical model depends on aims and problems which are to solve. As well as in each area of biomathematics we may distinguish two loose categories of glucose kinetics model: the "pure" model whose end is the understanding of phenomena, and the "applied " model directed to their control [2]. The greater part of models involves insulin-glucose interaction, which is approximated by predator (insulin) - prey (glucose) interaction term. However, such method leads rather to "pure" case of models, because the 
measurement of insulin concentration in the blood plasma are complicated and expensive and therefore the model identification procedure in (oupatient) clinical conditions is problematic. In addition, the mechanisms of glucose regulation have complex structure, especially in their counterregulatory part and therefore the glucose-insulin interaction gives the one aspect of the glucose levels kinetics only.

In this paper a mathematical model of the blood glucose level regulation mechanism is presented. It satisfies in some sense the requirements of consistency, simplicity and identifiability. This model can be used in selfmonitoring programs for the diabetics.

The users of such program could be patient, their nurses, physicians, endocrinologists and biomathematics. It might help to determine the parameters of patient's organism, to forecast glycaemia and glycosuria under known daily regime and injected insulin doses, and, on the other hand, to plain regime of the day and insulinotherapy.

The model proposed here is based on upper and lower euglycaemia level bounds concept. It means that in the healty human organism the activity of blood glucose level regulation mechanisms varies from the prevailing of the glucose production (the counterregulatory hormons activity) under the lower bound through the equilibrium of the glucose production and utilization processes between the lower and upper bound to the prevailing of the glucose utilization (insulin activity), when the blood glucose level increases over the upper bound. The core of diabetes mellitus phenomenon is a relative insufficiency or complete absence of the insulin activity, therefore, in the case of diabetes, glucose utilization is not activated even if the glucose level exceedes the upper bound of euglycaemia.

The general scheme of the glucose uptake in the organism, which is used in proposed mathematical model involves the following processes:

- external glucose (food) intake

- insulin independent glucose utilization

- renal threshold mechanism

- effect of the activity of the natural glycaemia regulation mechanisms (as described above)

- insulin dependent glucose utilization

- effect of injected insulin activity.

\section{THE SIMPLEST MATHEMATICAL MODEL}

Let $D_{l}, D_{0}$ and $D_{u}$ are the domains in $R^{1}$ :

$$
D_{l}=\left\{x ; \quad x \leq x_{l}\right\}, D_{0}=\left\{x ; \quad x_{l} \leq x \leq x_{u}\right\}, D_{u}=\left\{x ; \quad x \geq x_{u}\right\} .
$$

where $x_{l}, x_{u}, x_{l}<x_{u}$ - the lower and the upper bounds of the englycaemia, $x=x(t)-$ glycaemia level at the time moment $t$. 
Let us define a function $K(x)$ as follows:

$$
K(x)= \begin{cases}K_{l}>0, & x \in D_{l} \\ 0, & x \in D_{0} \\ K_{u}<0, & x \in D_{u}\end{cases}
$$

where $K_{l}$ and $K_{u}$ are constants.

Let us introduce the function $f_{1}(t)$, which describes the external glucose intake and the function $f_{2}(t)$, which is insulin independent glucose utilization term. Let $f(t)=f_{1}(t)-f_{2}(t)$.

Then we can describe glucose level dynamics as follows:

$$
\begin{aligned}
& \frac{d x}{d t} \quad+K_{l}\left(x-x_{l}\right)=f(t), \quad x \in D_{l}, \\
& \frac{d x}{d t}=f(t), \quad x \in D_{0}, \\
& \frac{d x}{d t} \quad+K_{u}\left(x-x_{u}\right)=f(t), \quad x \in D_{u},
\end{aligned}
$$

or, breafly,

$$
\frac{d x}{d t}+g(x)=f(t)
$$

where

$$
g(x)= \begin{cases}K_{l}\left(x-x_{l}\right), & x \in D_{l} \\ 0, & x \in D_{0} \\ K_{u}\left(x-x_{u}\right), & x \in D_{u}\end{cases}
$$

is a continuous Lipschitz function, therfore, (1) equation has a unique solution, which can be written in the following "pseudoanalytical" form:

$$
x(t)=\left(x_{0}+\int_{t_{0}}^{t}\left[K_{j} x_{j}+f(t)\right] e^{-K_{j} \tau} d \tau\right) e^{K_{j} t}, x \in D_{j}, j=l, 0, u .
$$

\section{THE MODEL IDENTIFICATION AND IMPROVING PROB- LEMS}

There are 4 parameters in the (1) model to be identified: $K_{l}, K_{u}, x_{l}, x_{u}$. That can be done by least square method, involving some regularization algoritms (see [1]).

The model can be improved by involving of glucosuria term. Then the main equation of the model becomes as follows:

$$
\frac{d x}{d t}+g(x)+q(x)=f(t) .
$$


where $q(x)$ is a renal theshold function, which can be expressed in such way:

$$
q(x)= \begin{cases}0, & x<x_{g 1} \\ Q\left(x-x_{g 1}\right), & x_{g 1} \leq x \leq x_{g 2} \\ Q\left(x_{g 2}-x_{g 1}\right), & x \leq x_{g 2}\end{cases}
$$

here $Q, x_{g 1}, x_{g 2}$ are some constants, which chacterize the renal threshold functions [1], [3]. Denote, that parameters of the function $q(x)$ can by identified independent from the other parameters of (1) equation.

\section{REFERENCES}

[1] Čiegis R., Meilūnas M., Some algorithms in mathematical modelling of Diabetes mellitus, Informatica, 6 (1995), P. 15 -33.

[2] Švitra D., Dynamics of Physiological Systems, Vilnius: Mokslas, (1989) (in Russian).

[3] Schueck O., Functional investigation of the renal, Prague: Avicenum, (1981) (in Russian).

[4] Diabetes, Nutrition \& Metabolism, 4 (1), (1991)

[5] Olson O.Ch., Diagnosis and Management of Diabetes Mellitus, New York: Raven Press, (1989) 